\title{
Palm pairs and the general mass-transport principle
}

\author{
Daniel Gentner and Günter Last*
}

January 31, 2009

\begin{abstract}
We consider a lcsc group $G$ acting properly on a Borel space $S$ and measurably on an underlying $\sigma$-finite measure space. Our first main result is a transport formula connecting the Palm pairs of jointly stationary random measures on $S$. A key (and new) technical result is a measurable disintegration of the Haar measure on $G$ along the orbits. The second main result is an intrinsic characterization of the Palm pairs of a $G$-invariant random measure. We then proceed with deriving a general version of the mass-transport principle for possibly non-transitive and non-unimodular group operations first in a deterministic and then in its full probabilistic form.
\end{abstract}

Keywords: random measure, Palm measure, stationarity, invariance, locally compact group, operation, Haar measure, orbit, mass-transport principle

2000 Mathematics Subject Classification: Primary 60D05, 60G55; Secondary 60G60

\section{Introduction}

Let $G$ be a locally compact second countable Hausdorff (in short lcsc) topological group operating properly on a Borel space $(S, \mathcal{S})$. Consider a $\sigma$-finite measure $M$ on $S \times S$ that is invariant under joint shifts of both arguments. It is helpful to think of $M(C \times D)$ as the amount of mass transported from $C \in \mathcal{S}$ to $D \in \mathcal{S}$. Assume first that the group is unimodular and that the group action is transitive, i.e. that there is only one orbit. If $B \in \mathcal{S}$ has positive and finite invariant measure, then

$$
M(B \times S)=M(S \times B) .
$$

This mass-transport principle [2,3] plays an important role in the study of percolation on graphs. Häggström [7] was the first who has used it for this purpose. Last and Thorisson [13] noticed that (1.1) can also be seen as a special case of Neveu's classical exchange formula [18]. The exchange formula is a versatile tool in the theory of random measures and point processes [1]. A general lcsc group admits a modular function $\Delta: G \rightarrow(0, \infty)$

\footnotetext{
${ }^{*}$ Postal address: Institut für Stochastik, Universität Karlsruhe (TH), 76128 Karlsruhe, Germany. Email addresses: daniel.gentner@kit.edu, last@math.uni-karlsruhe.de
} 
satisfying (2.1) below. Still assuming the group action to be transitive, (1.1) generalizes to

$$
\int \tilde{\Delta}(s, t) \mathbf{1}_{B}(s) M(d(s, t))=\int \mathbf{1}_{B}(t) M(d(s, t)) .
$$

where $\tilde{\Delta}(s, t):=\Delta(g)$ if $g s=t$. This formula can be derived from Theorem 4.4 in [11]. One purpose of this paper is to establish the mass-transport principle (1.2) in the general, possibly non-transitive and non-unimodular case. This principle holds for all $B$ satisfying a natural symmetry condition and with a suitably generalized definition of the function $\tilde{\Delta}(s, t)$, see Theorem 6.8. Equation (1.2) is just a special case of a more general transport formula for stationary random measures on $S$, see Theorems 4.1 and 6.3. The main aim of this paper is to derive these and a series of related results in our general setting described above.

In probability theory stationarity refers to invariance of the distribution under the shifts induced by $G$. In this paper we will express stationarity by assuming that $G$ operates measurably on an underlying $\sigma$-finite measure space $(\Omega, \mathcal{A}, \mathbb{P})$, where $\mathbb{P}$ is invariant under $G$. (We will use a probabilistic language even though $\mathbb{P}$ is not assumed to have the finite total mass 1.) A stationary random measure $\xi$ is then just a $\sigma$-finite kernel from $\Omega$ to $S$ which is invariant under joint shifts. We will use this terminology also for other kernels. Fundamental objects associated with an invariant random measure $\xi$ are its Palm pairs $(\nu, Q)$, where $\nu$ is a supporting measure of $\xi$ (a $\sigma$-finite measure on $S$ equivalent to $\mathbb{E}_{\mathbb{P}} \xi(\cdot)$ ) and $Q$ is an appropriate kernel from $S$ to $\Omega$ disintegrating the Campbell measure of $\xi$, see (3.1). Given $\nu$, such a kernel exists under weak technical assumptions, see Kallenberg [10]. In the transitive situation $(G s=S$ for all $s \in S$ ) the Palm kernel can be obtained from a single measure on $(\Omega, \mathcal{A})$, the Palm measure of $\xi$, by suitable shifts, see e.g. [21] (treating the case of a group acting on itself) and [19, 10, 12]. The seminal paper Mecke [16] is dealing with an Abelian group operating on itself. This case is of particular relevance for applications of Palm theory, see e.g. [4, 20]. A very general approach to Palm measures and their invariance properties is taken in Kallenberg [10]. Much of the notation and terminology of the present paper stems from this source.

The paper starts with a brief repetition of the basic terminology for invariant measures and kernels (Section 2) and for random measures and their Palm pairs (Section 3). A key technical result is a measurable and invariant disintegration of the Haar measure on $G$ along the orbits in $S$, Theorem 2.1. The first main result is a transport formula connecting the Palm pairs of two jointly stationary random measures $\xi$ and $\eta$ on $S$, Theorem 4.1. This result extends Theorem 3.6 in [13] to the more general case studied in this paper. Corollary 4.2 generalizes Neveu's well-known exchange formula (see e.g. [18]). Our second main result is an intrinsic characterization of Palm pairs of an invariant random measure, Theorem 5.1. This extends Mecke's [16] famous characterization of Palm measures of stationary random measures on an Abelian group (cf. also [11] for the case of a general group and [19], [12] for the case of a homogeneous space). We then proceed in Section 6 with deriving a general version of the mass-transport principle (1.2) first in a deterministic form (Subsection 6.1) and then in its full probabilistic form (Subsection 6.2). Theorem 6.3 is the third main result of this paper. Applications of this principle to partially stationary tessellations of manifolds will be provided in the paper [6], which is in preparation.

Finally in this introduction we state our basic notation for measures and kernels. Let 
$(T, \mathcal{T})$ denote a measurable space. If $\mu$ is a measure on $(T, \mathcal{T})$ and $f: T \rightarrow[-\infty, \infty]$ is measurable then we denote the integral $\int f d \mu$ by $\mu f \equiv \mu(f)$ whenever it is well-defined. We denote by $\mathcal{T}_{+}$the space of $\mathcal{T}$-measurable $[0, \infty]$-valued functions. For $f \in \mathcal{T}_{+}$we write $f \cdot \mu$ for the measure $A \mapsto \int \mathbf{1}_{A}(t) f(t) \mu(d t)$. If $\left(T^{\prime}, \mathcal{T}^{\prime}\right)$ is another measurable space then a kernel $\kappa$ from $T$ to $T^{\prime}$ is a map $\kappa: T \times \mathcal{T}^{\prime} \rightarrow[0, \infty]$ such that for each $B \in \mathcal{T}^{\prime}$ the map $t \mapsto \kappa(t, B)$ is measurable and that for each $t \in T$ the setfunction $\mu(t, \cdot)$ is a measure on $T^{\prime}$. A kernel from $T$ to $T$ is usually refered to as a kernel on $T$. The kernel $K$ is $\sigma$-finite if for each $t \in T$ the measure $K(t, \cdot)$ is $\sigma$-finite. In this paper all kernels are assumed to be $\sigma$-finite. Let $K$ be a kernel from $(T, \mathcal{T})$ to another measurable space $\left(T^{\prime}, \mathcal{T}^{\prime}\right)$. If $\mu$ is a measure on $(T, \mathcal{T})$ then $\mu \otimes K$ denotes the measure on the product space $\left(T \times T^{\prime}, \mathcal{T} \otimes \mathcal{T}^{\prime}\right)$ defined by $\mu \otimes K(A)=\iint \mathbf{1}_{A}(s, t) K(s, d t) \mu(d s)$, where $\mathbf{1}_{A}$ is the indicator function of $A \in \mathcal{T} \otimes \mathcal{T}^{\prime}$. (Note that $\sigma$-finiteness of $K$ implies measurability of $s \mapsto \int \mathbf{1}_{A}(s, t) K(s, d t)$ in a similar way as in standard proofs of Fubini's theorem.)

\section{Invariant measures and disintegrations}

Let $G$ be a locally compact second countable Hausdorff (in short lcsc) topological (multiplicative) group with unit element $e$. The group $G$ is equipped with the Borel $\sigma$-field $\mathcal{G}$. Elements of $G$ will usually be denoted by $g$ or $h$. We fix a left-invariant locally finite Haar measure $\lambda$ on $G$. Left-invariance means

$$
\int f(h g) \lambda(d g)=\int f(g) \lambda(d g), \quad h \in G, f \in \mathcal{G}_{+} .
$$

The modular function is a continuous homomorphism $\Delta: G \rightarrow(0, \infty)$ satisfying

$$
\int f(g h) \lambda(d g)=\Delta\left(h^{-1}\right) \int f(g) \lambda(d g), \quad h \in G,
$$

for all $f \in \mathcal{S}_{+}$. This modular function has the property

$$
\int f\left(g^{-1}\right) \lambda(d g)=\int \Delta\left(g^{-1}\right) f(g) \lambda(d g), \quad f \in \mathcal{G}_{+} .
$$

The group $G$ is called unimodular if $\Delta(g)=1$ for all $g \in G$. By (2.1) $G$ is unimodular if and only if $\lambda$ is right-invariant.

Let $(S, \mathcal{S})$ be a Borel space. Elements of $S$ will be named $s$ or $t$. We assume that $G$ operates on $S$, i.e. we assume that there is a mapping $(g, s) \mapsto g s$ from $G \times S$ to $S$ having $g(h s)=(g h) s$ and $e s=s$ for all $g, h \in G$ and $s \in G$. Here $e$ denotes the neutral element of $G$. The projections $\pi_{s}: G \rightarrow S, s \in S$, and the translations $\theta_{g}: S \rightarrow S, g \in G$, are given by

$$
\pi_{s}(g)=\theta_{g}(s)=g s, \quad g \in G, s \in S .
$$

The trace of $G$ in $S$ starting in $s \in S$, i.e. the set $\pi_{s}(G)=G s$ is called the orbit of $s$. We assume that $G$ operates properly on $S$ in the sense that the operation is measurable as a map $G \times S \rightarrow S$ and that the set of all pushforwards $\mu_{s}:=\lambda \circ \pi_{s}^{-1}, s \in S$, of the Haar measure under the projections is uniformly $\sigma$-finite. This means we require the existence of a measurable partition $B_{1}, B_{2}, \ldots$ of $S$ such that $\mu_{s}\left(B_{n}\right)<\infty, s \in S, n \in \mathbb{N}$. 
This concept was introduced by Kallenberg in [10] and generalizes the classical notion of a topologically proper (continuous) operation of a lcsc group on a lcsc space. He also showed ([10], Lemma 2.1) that properness is equivalent to the existence of a measurable function $k: S \rightarrow(0, \infty)$ such that

$$
\mu_{s} k=\int k(t) \mu_{s}(d t)<\infty, \quad s \in S
$$

Denote the cosets of the stabilizers as

$$
G_{s, t}:=\{g \in G: g s=t\}=\pi_{s}^{-1}(\{t\}), \quad s, t \in S,
$$

which are measurable sets in $G$ under our assumptions on $S$. We introduce a kernel $\kappa$ from $S \times S$ to $G$ that enables us to handle stabilizers and their cosets in $G$ within integral equations. This kernel satisfies

$$
\int f(g s, g) \lambda(d g)=\iint f(t, g) \kappa_{s, t}(d g) \mu_{s}(d t), \quad f \in(\mathcal{S} \otimes \mathcal{G})_{+}, s \in S
$$

In particular $\kappa$ disintegrates the Haar measure $\lambda$ on $G$ along each orbit via

$$
\int f(g) \lambda(d g)=\iint f(g) \kappa_{s, t}(d g) \mu_{s}(d t), \quad f \in \mathcal{G}_{+}, s \in S .
$$

The next theorem will be proved in the Appendix.

Theorem 2.1. There is a kernel $\kappa$ from $S \times S$ to $G$ with the disintegration property (2.4) (and in particular (2.5)) and the following properties:

(i) $\kappa_{s, g t}=\kappa_{s, t} \circ \theta_{g}^{-1}, \quad g \in G, s, t \in S$,

(ii) $\kappa_{s, t}$ is concentrated on $G_{s, t}:=\{g \in G: g s=t\}$ for $t \in G s, s \in S$,

(iii) $\kappa_{s, t}(G)=1, \quad t \in G s, s \in S$.

A kernel $\kappa$ with the above properties will be fixed throughout the paper.

Example 2.2. Assume that $G$ operates transitively on $S$, i.e. that there is only one orbit. Fix some $c \in S$. By (2.6) below the measures $\mu_{s}, s \in S$, are all multiples of $\mu_{c}$. By Corollary 2.6 in [10] $\mu_{c}$ is up to normalization the unique invariant $\sigma$-finite measure on $S$.

The kernel $\kappa$ can be constructed by a suitable translation of the probability measure $\kappa_{c}:=\kappa_{c, c}$. Indeed, let $s \in S$ and take some $g_{s} \in G_{c, s}$, i.e. $g_{s} c=s$. Then $G_{s, s}=g_{s} G_{c, c} g_{s}^{-1}$ and it is easy to see that $\int \mathbf{1}\left\{g_{s} g g_{s}^{-1} \in \cdot\right\} \kappa_{c}(d g)$ is a left-invariant measure on $G_{s, s}$. If $G_{s, s}$ is compact, then this measure must coincide with $\kappa_{s, s}$, see also Corollary 7.3. Now take $t \in S$ and some $g \in G_{s, t}$. By Theorem 2.1 (ii) we then have $\kappa_{s, t}=\kappa_{s, s} \circ \theta_{g}^{-1}$.

Example 2.3. We may further specialize Example 2.2 by assuming that $S=G$ and that $(g, s) \mapsto g s$ is just the multiplication in the group. Then $\mu_{s}=\Delta\left(s^{-1}\right) \lambda$ for all $s \in S$. For $s, t \in G$ we have $G_{s, t}=\left\{t s^{-1}\right\}$, while $\kappa_{s, t}$ is the Dirac measure located at $t s^{-1}$. 
A measure $\nu$ on $S$ is invariant (or G-invariant) if

$$
\nu \circ \theta_{g}=\nu, \quad g \in G .
$$

The projection measures $\mu_{s}, s \in S$, are clearly invariant measures on $S$. They have the additional property that

$$
\mu_{g s}=\Delta\left(g^{-1}\right) \mu_{s}, \quad g \in G, s \in S,
$$

which means that the properness condition upon the operation enforces

$$
\Delta(g)=1, \quad g \in G_{s, s}, s \in S .
$$

Hence the measures

$$
\varphi_{s}:=\frac{\mu_{s}}{\mu_{s} k}, \quad s \in S,
$$

are invariant, uniformly normalized in the sense that $\varphi_{s} k=1, s \in S$, and even constant on orbits, i.e.

$$
\varphi_{g s}=\varphi_{s}, \quad s \in S, g \in G .
$$

By Fubinis theorem $\varphi$ is a kernel on $S$. Kallenberg proved in [10] that this kernel can be used as a normalized extremal generator of the convex cone of all $\sigma$-finite invariant measures on $S$ since for any $\sigma$-finite invariant measure $\nu$ on $S$

$$
\nu(\cdot)=\int \varphi_{s}(\cdot) k(s) \nu(d s)
$$

cf. Theorem 2.4 in [10]. Choosing a system $O$ of representatives of the orbits $G s, s \in S$, the space $S$ splits into the disjoint union

$$
S=\bigcup_{b \in O} G b
$$

and we may consider the choice function $\beta: S \rightarrow S$ defined by $\beta(s):=b_{s}, b_{s}$ denoting the previously chosen representative of $G s$. A fixed choice of a system of representatives allows for the following canonical transfer of the modular function from $G$ to $S$

$$
\Delta^{*}(s):=\Delta\left(g_{s}^{-1}\right), \quad s \in S,
$$

where $g_{s} \in G_{\beta(s), s}$. This definition is independent of the choice of $g_{s}$. Indeed, if $g, h \in$ $G_{\beta(s), s}$ then $g^{-1} h \in G_{\beta(s), \beta(s)}$ so that $(2.7)$ implies $1=\Delta\left(g^{-1} h\right)$, i.e. $\Delta\left(g^{-1}\right)=\Delta\left(h^{-1}\right)$.

Properness imposes natural restrictions upon the size of the stabilizers and thus affects the relative size of the orbits. Since in the case of countable $S$ these can be seen most easily and since this case is of independent interest for applications (e.g. for percolation on countable graphs) we investigate what properness means in this situation. The cardinality of a set $A$ is denoted by $|A|$. 
Lemma 2.4. $G$ operates properly on a countable set $S$ if and only if

$$
0<\lambda\left(G_{s, s}\right)<\infty, \quad s \in S
$$

In this case

$$
\Delta^{*}(s)=\frac{\lambda\left(G_{s, s}\right)}{\lambda\left(G_{\beta(s), \beta(s)}\right)}=\frac{\left|G_{s, s} \beta(s)\right|}{\left|G_{\beta(s), \beta(s)} s\right|}, \quad s \in S,
$$

and either all orbits are infinite or all orbits are finite.

Proof. Countability implies $0<\lambda\left(G_{s, s}\right), s \in S$, since $\lambda\left(G_{s, s}\right)=0$ for some $s$ implies $\lambda(G)=\sum_{t \in G s} \lambda\left(G_{s, t}\right)=0$ by left-invariance of $\lambda$ - an impossibility. For any $k$ on $S$ we have

$$
\mu_{s} k=\int k(g s) \sum_{t \in G s} \mathbf{1}\{g s=t\} \lambda(d g)=\lambda\left(G_{s, s}\right) \sum_{t \in G s} k(t), \quad s \in S .
$$

Hence if the operation is proper then $\lambda\left(G_{s, s}\right)<\infty$ for any $s$. This equation also shows the converse since we may always choose $k>0$ on $S$ such that $\sum_{t \in G s} k(t)<\infty, s \in S$. Now choose $k>0$ on $S$ such that $\sum_{s \in G b} k(s)<\infty, b \in O$, and even $\sum_{s \in G b} k(s)=\sum_{s \in G b^{\prime}} k(s)$, $b, b^{\prime} \in O$. Then

$$
\Delta^{*}(s)=\frac{\mu_{s} k}{\mu_{\beta(s)} k}=\frac{\lambda\left(G_{s, s}\right) \sum_{t \in G s} k(t)}{\lambda\left(G_{\beta(s), \beta(s)}\right) \sum_{t \in G \beta(s)} k(t)}=\frac{\lambda\left(G_{s, s}\right)}{\lambda\left(G_{\beta(s), \beta(s)}\right)}, \quad s \in S .
$$

By left-invariance of Haar measure and elementary properties of left cosets

$$
\frac{\lambda\left(G_{s, s}\right)}{\lambda\left(G_{s, s} \cap G_{t, t}\right)}=\left[G_{s, s}: G_{s, s} \cap G_{t, t}\right]=\left|G_{s, s} t\right|, \quad s, t \in S
$$

where $[G: H]$ denotes the left index of a subgroup $H \subset G$, i.e. the number of the left cosets of $H$. Hence

$$
\Delta^{*}(s)=\frac{\left|G_{s, s} \beta(s)\right|}{\left|G_{\beta(s), \beta(s)} s\right|}, \quad s \in S .
$$

For any orbit $G s$ we have $\lambda(G)=|G s| \lambda\left(G_{s, s}\right)$ and hence if $|G t|=\infty$ for some $t \in S$, then necessarily $\lambda(G)=\infty$ and thus for any other orbit $G s$ also $|G s|=\infty$ by properness.

If $S$ is not countable we need to establish measurability of $\beta$ and $\Delta$. For this recall the concept of universal measurability. If $\mu$ is a measure on $(S, \mathcal{S})$ then $\mathcal{S}^{\mu}$ denotes the completion of $\mathcal{S}$ with respect to $\mu$. The universal completion of a $\sigma$-algebra $\mathcal{S}$ is then defined as

$$
\mathcal{S}^{u}=\bigcap_{\mu} \mathcal{S}^{\mu}
$$

where the intersection is taken over the class of all finite measures (or simply over the class of probability measures) on $(S, \mathcal{S})$. Its elements are called universally measurable sets and a map $f: S \rightarrow T$ is called universally measurable if it is $\mathcal{S}^{u} / \mathcal{T}$-measurable. 
Lemma 2.5. The following holds:

(i) The orbits $G s, s \in S$, are universally measurable sets in $S$;

(ii) If $O \in \mathcal{S}$ then $\beta$ is universally measurable;

(iii) If $O \in \mathcal{S}$ then for any $B \in \mathcal{G}$ the map $s \mapsto \kappa_{\beta(s), s}(B)$ is universally measurable;

(iv) If $O \in \mathcal{S}$ then $\Delta^{*}$ is universally measurable.

Proof. Consider the Borel isomorphism $\psi: G \times S \rightarrow G \times S$ given by $\psi(g, s)=(g, g s)$ and the measurable sets $A_{s}:=\psi(G \times\{s\}), s \in S$. Since $G$ is Borel the projection of $A_{s}$ on $S$ is a universally measurable set in $S$ according to Dellacherie and Meyer [5] Section III..44. These projections are clearly the orbits of the operation, hence $(i)$ follows.

For $(i i)$ note that for $B \subset S$ we have $\beta^{-1}(B)=G(B \cap O)$. Hence $\beta^{-1}(B)=G(B \cap O)=$ $\operatorname{pr}_{S}(\varphi(G \times(B \cap O)))$ and for $B, O \in \mathcal{S}$ this implies that $\beta^{-1}(B)$ is universally measurable since $G$ is Borel.

(iii) holds since the map $(s, t) \mapsto \kappa_{s, t}(B)$ is measurable according to Theorem 2.1 while $s \mapsto(\beta(s), s)$ is universally measurable by $(i i)$ and elementary properties of the product $\sigma$-algebra.

The universal measurability of $\Delta^{*}$ and hence $(i v)$ follows from the representation

$$
\Delta^{*}(s)=\int \Delta\left(g^{-1}\right) \kappa_{\beta(s), s}(d g), \quad s \in S .
$$

Remark 2.6. The concept of universal measurability is useful when dealing with finite or at least $\sigma$-finite measures $\mu$ on a measurable space $(S, \mathcal{S})$ since for a universally measurable map $f$ integrals $\mu f$ with respect to any such $\mu$ make sense. Any $\sigma$-finite $\mu$ has a unique extension to the class of $\mathcal{S}^{\mu}$-measurable functions and in particular to the (smaller) class of $\mathcal{S}^{u}$-measurable functions. We make heavy use of this fact for almost all results in this paper without further notice.

Throughout this paper we fix one system $O$ of representatives of the orbits and require $O \in \mathcal{S}$ such that $\beta$ and $\Delta^{*}$ are universally measurable by Lemma 2.5. By means of (2.8) and $\beta$ equation (2.9) can be modified as follows:

$$
\nu(\cdot)=\int \varphi_{s}(\cdot) k(s) \nu(d s)=\int \mu_{\beta(s)}(\cdot) \mu_{\beta(s)}(k)^{-1} k(s) \nu(d s)=\int \mu_{b}(\cdot) \nu^{*}(d b),
$$

where $\nu^{*}=\left(\mu_{\beta}(k)^{-1} k \cdot \nu\right) \circ \beta^{-1}$ is a measure concentrated on $O$, in the sense that any measurable set $B$ in $S$ being disjoint with $O$ has $\nu^{*}(B)=0$.

\section{Random measures and Palm pairs}

As before let $(S, \mathcal{S})$ be a Borel space. Let $\mathbf{M}(S)$ denote the space of all $\sigma$-finite measures on $S$. We equip $\mathbf{M}(S)$ with the smallest $\sigma$-field $\mathcal{M}(S)$ making the mappings $\mu \mapsto \mu(B)$ for all $A \in \mathcal{S}$ measurable. Let $(\Omega, \mathcal{A}, \mathbb{P})$ be a $\sigma$-finite measure space. We use a probabilistic language even though $\mathbb{P}$ need not be a probability measure. A random measure on $S$ is a measurable mapping $\xi: \Omega \rightarrow \mathbf{M}(S)$ that is uniformly $\sigma$-finite in the sense that there is 
a partition $B_{1}, B_{2}, \ldots$ of $S$ such that $\xi\left(B_{i}\right)<\infty \mathbb{P}$-a.e. for any $i \in \mathbb{N}$. We use the kernel notation $\xi(\omega, B):=\xi(\omega)(B)$. If $\xi$ is a random measure on $S$ then the Campbell measure $C_{\xi}$ of $\xi$ (with respect to $\mathbb{P}$ ) is the measure on $\Omega \times S$ satisfying

$$
C_{\xi} f=\iint f(\omega, s) \xi(\omega, d s) \mathbb{P}(d \omega), \quad f \in(\mathcal{A} \otimes \mathcal{S})_{+} .
$$

This measure is finite on sets of the form $\left\{\omega \in A: \xi\left(\omega, B_{i}\right) \leq n\right\} \times B_{i}$, where $i, n \in \mathbb{N}$, $A \in \mathcal{A}$ has $\mathbb{P}(A)<\infty$ and the $B_{i}$ are as in the definition of $\xi$. It follows that $C_{\xi}$ is $\sigma$-finite. Hence there exists a supporting measure of $\xi$, i.e. a $\sigma$-finite measure $\nu$ on $S$ such that $C_{\xi}(\Omega \times \cdot)$ and $\nu$ are equivalent in the sense of mutual absolute continuity. If $(\Omega, \mathcal{A})$ is Borel, then there is a $\sigma$-finite kernel $Q$ from $S$ to $\Omega$ disintegrating $C_{\xi}$ as follows:

$$
C_{\xi} f=\iint f(\omega, s) Q_{s}(d \omega) \nu(d s), \quad f \in(\mathcal{A} \otimes \mathcal{S})_{+} .
$$

We call a pair $(\nu, Q)$ satisfying (3.1) a Palm pair of $\xi$. The kernel $Q$ is the $\nu$-associated Palm kernel of $\xi$. To make the dependence on $\xi$ (but not on $\mathbb{P}$ ) explicit, we often write $\left(\nu_{\xi}, Q_{\xi}\right):=(\nu, Q)$.

We now return to the setting established in Section 2. In addition we assume that $G$ is operating measurably on $\Omega$. There is no risk of confusion to denote the associated translations by $\theta_{g}: \Omega \rightarrow \Omega, g \in G$. The set $\left\{\theta_{g}\right\}_{g \in G}$ is commonly refered to as a flow on $\Omega$. Our basic assumption is that $\mathbb{P}$ is invariant under the flow, i.e.

$$
\mathbb{P} \circ \theta_{g}^{-1}=\mathbb{P}, \quad g \in G .
$$

A random measure $\xi$ on $S$ is called invariant (or $G$-invariant) if it satisfies

$$
\xi\left(\theta_{g} \omega, B\right)=\xi\left(\omega, \theta_{g}^{-1} B\right), \quad g \in G, \omega \in \Omega, B \in \mathcal{S} .
$$

Similarly a kernel $Q$ from $S$ to $\Omega$ is called invariant if

$$
Q(g s, A)=Q\left(s, g^{-1} A\right), \quad g \in G, s \in S, A \in \mathcal{A} .
$$

Assume that $\xi$ is an invariant random measure. Then it is easy to see that the Campbell measure of $\xi$ is (jointly) invariant, i.e. invariant under joint shifts of both arguments, see the Appendix for an explicit definition. If $(\Omega, \mathcal{A})$ is Borel, Corollary 3.5 in [10] implies that there is an invariant Palm pair $(\nu, Q)$ of $\xi$, meaning that both $\nu$ and $Q$ are invariant. Next we formulate the refined Campbell theorem. Although a simple consequence of the definitions, it is the main tool of Palm calculus for invariant random measures. Recall the representation (2.13) and that $\theta_{e}$ is the identity on $\Omega$. Adapting common terminology of probability theory, we denote integration with respect to a measure $Q^{\prime}$ on $\Omega$ by $\mathbb{E}_{Q^{\prime}}$.

Proposition 3.1. Assume that the invariant random measure $\xi$ has an invariant Palm pair $(\nu, Q)$. Then, for all $f \in(\mathcal{A} \otimes \mathcal{G} \otimes \mathcal{S})_{+}$,

$$
\mathbb{E}_{\mathbb{P}} \iint f\left(\theta_{g}^{-1}, g, \beta(t)\right) \kappa_{\beta(t), t}(d g) \xi(d t)=\int \mathbb{E}_{Q_{b}} \int f\left(\theta_{e}, g, b\right) \lambda(d g) \nu^{*}(d b) .
$$


Proof. Let $f \in(\mathcal{A} \otimes \mathcal{G} \otimes \mathcal{S})_{+}$and denote the right-hand side of (3.5) by I. By (2.5) and Fubini's theorem,

$$
I=\iint \mathbb{E}_{Q_{b}} \int f\left(\theta_{e}, g, b\right) \kappa_{b, t}(d g) \mu_{b}(d t) \nu^{*}(d b) .
$$

By Theorem 2.1 (ii), $\kappa_{b, t}$ is concentrated on $G_{b, t}$. Hence we obtain by invariance (3.4) of the Palm kernel that

$$
I=\iint \mathbb{E}_{Q_{t}} \int f\left(\theta_{g}^{-1}, g, b\right) \kappa_{b, t}(d g) \mu_{b}(d t) \nu^{*}(d b) .
$$

For $b \in O=\beta(S)$ and $t \in G b$ we have $b=\beta(t)$. Since $\nu^{*}$ is concentrated on $O$ we obtain

$$
I=\iint \mathbb{E}_{Q_{t}} \int f\left(\theta_{g}^{-1}, g, \beta(t)\right) \kappa_{\beta(t), t}(d g) \mu_{b}(d t) \nu^{*}(d b) .
$$

An application of (2.13) yields

$$
I=\int \mathbb{E}_{Q_{t}} \int f\left(\theta_{g}^{-1}, g, \beta(t)\right) \kappa_{\beta(t), t}(d g) \nu(d t) .
$$

The defining property (3.1) of a Palm pair yields the assertion (3.5).

Remark 3.2. Assume that $G=\{e\}$. Then $\lambda=\delta_{e}, O=S, \mu_{s}=\delta_{s}$ and $\kappa_{s, t}=\mathbf{1}\{s=t\} \delta_{e}$. Let $\xi$ be a random measure on $S$ with supporting measure $\nu$. Then $\xi$ is invariant. Let $(\nu, Q)$ be a Palm pair of $\xi$. Then $(\nu, Q)$ is invariant, $\nu^{*}=\nu$, and the refined Campbell theorem (3.5) boils down to the defining equation (3.1) of a Palm pair. Hence general random measures can be treated within our framework of invariant random measures.

Example 3.3. Consider the situation of Example 2.2, i.e. assume that $G$ operates transitively on $S$. Let $\xi$ be an invariant random measure on $S$. Fixing some $c \in S$, we can take $\nu:=\mu_{c}$ as a supporting measure of $\xi$. Moreover, taking $\beta \equiv c$, we clearly have $\nu^{*}=\delta_{c}$. Then (3.5) simplifies to

$$
\mathbb{E}_{\mathbb{P}} \iint f\left(\theta_{g}^{-1}, g\right) \kappa_{c, t}(d g) \xi(d t)=\mathbb{E}_{Q_{c}} \int f\left(\theta_{e}, g\right) \lambda(d g), \quad f \in(\mathcal{A} \otimes \mathcal{G})_{+} .
$$

In particular,

$$
Q_{c}(A)=\mathbb{E}_{\mathbb{P}} \iint \mathbf{1}_{A}\left(\theta_{g}^{-1}\right) w(t) \kappa_{c, t}(d g) \xi(d t), \quad A \in \mathcal{A},
$$

where $w \in \mathcal{S}_{+}$has $\int w d \mu_{c}=1$. In fact one can use (3.7) to give an explicit definition of $Q_{c}$ (without any Borel assumption on $\Omega$ ) and then derive (3.6). This is the approach taken in [19] and [12]. In the further special case $S=G$ of Example 2.3 we may take $c=e$ and (3.6) simplifies to

$$
\mathbb{E}_{\mathbb{P}} \int f\left(\theta_{g}^{-1}, g\right) \xi(d g)=\mathbb{E}_{Q_{e}} \int f\left(\theta_{e}, g\right) \lambda(d g), \quad f \in(\mathcal{A} \otimes \mathcal{G})_{+} .
$$

Equation (3.7) changes accordingly. This skew factorization is the standard approach to Palm measures of stationary random measures on a group. We refer to [10] for more details and historical remarks. 
Example 3.4. Assume that $O$ is finite, i.e. that there are only finitely many orbits. Following [2] we call this the quasi-transitive case. Let $\xi$ be an invariant random measure on $S$. Then without loss of generality a supporting measure may be chosen as $\nu:=$ $\sum_{b \in O^{\prime}} \mu_{b}$ for some $O^{\prime} \subset O$. It is easy to check that $\nu^{*}=\sum_{b \in O^{\prime}} \delta_{b}$. Then if $\mathrm{Q}$ is an associated invariant Palm kernel of $\xi$ the refined Campbell theorem (3.5) now implies

$$
\mathbb{E}_{\mathbb{P}} \iint f\left(\theta_{g}^{-1}, g\right) \kappa_{b, t}(d g) \xi_{b}(d t)=\mathbb{E}_{Q_{b}} \int f\left(\theta_{e}, g\right) \lambda(d g), \quad f \in(\mathcal{A} \otimes \mathcal{G})_{+}, b \in O^{\prime},
$$

where $\xi_{b}$ is the restriction of $\xi$ to the orbit $G b$. Let $b \in O^{\prime}$ and $w_{b} \in \mathcal{S}_{+}$with $\int w_{b} d \mu_{b}=1$. Then (3.9) implies

$$
Q_{b}(A)=\mathbb{E}_{\mathbb{P}} \iint \mathbf{1}_{A}\left(\theta_{g}^{-1}\right) w_{b}(t) \kappa_{b, t}(d g) \xi_{b}(d t), \quad A \in \mathcal{A} .
$$

This can be used for defining the Palm kernel $Q$ explicitly, just as in the transitive case of Example 3.3.

\section{The transport formula}

In the remainder of the paper we assume that the lcsc group $G$ operates measurably on $(\Omega, \mathcal{A})$ and properly on the Borel space $(S, \mathcal{S})$. In this section we fix an invariant $\sigma$-finite measure $\mathbb{P}$ on $(\Omega, \mathcal{A})$. Our aim is to derive a fundamental transport property of Palm measures. In the special case where $G=S$ is an Abelian group the result boils down to Theorem 3.6 in [13]. Other special cases will be discussed below. We use the function $\Delta^{*}$ defined by (2.10). A kernel $\gamma$ from $\Omega \times S$ to $S$ is called invariant if

$$
\gamma\left(\theta_{g} \omega, g s, B\right)=\gamma\left(\omega, s, g^{-1} B\right), \quad g \in G, s \in S, \omega \in \Omega, B \in \mathcal{S} .
$$

Theorem 4.1. Consider two invariant random measures $\xi$ and $\eta$ on $S$ and let $\gamma$ and $\delta$ be invariant kernels from $\Omega \times S$ to $S$ satisfying

$$
\iint \mathbf{1}\{(s, t) \in \cdot\} \gamma(\omega, s, d t) \xi(\omega, d s)=\iint \mathbf{1}\{(s, t) \in \cdot\} \delta(\omega, t, d s) \eta(\omega, d t)
$$

for $\mathbb{P}$-a.e. $\omega \in \Omega$ and $\left(\nu_{\xi}, Q_{\xi}\right),\left(\nu_{\eta}, Q_{\eta}\right)$ be invariant Palm pairs of $\xi$ and $\eta$ respectively. Then we have for any measurable function $f \in(\mathcal{A} \otimes \mathcal{G} \otimes \mathcal{S} \otimes \mathcal{S})_{+}$that

$$
\begin{aligned}
\int \mathbb{E}_{Q_{\eta, b}} \iint f\left(\theta_{g}^{-1}, g^{-1}, b, \beta(s)\right) \Delta^{*}(s) \kappa_{\beta(s), s}(d g) \delta(b, d s) \nu_{\eta}^{*}(d b) \\
=\int \mathbb{E}_{Q_{\xi, b}} \iint f\left(\theta_{e}, g, \beta(s), b\right) \kappa_{\beta(s), s}(d g) \gamma(b, d s) \nu_{\xi}^{*}(d b) .
\end{aligned}
$$

Proof. Let $k>0$ be as in (2.3). Then for any $b \in O=\beta(S)$ and $g \in G$

$$
l(b) \int k\left(g^{-1} h b\right) \lambda(d h)=1
$$


where $l(b):=\mu_{b}(k)^{-1}$. Take $f \in(\mathcal{A} \otimes \mathcal{G} \otimes \mathcal{S} \otimes \mathcal{S})_{+}$and denote the right-hand side of (4.3) by $I$. By Fubini's theorem,

$$
I=\int \mathbb{E}_{Q_{\xi, b}} \iiint f\left(\theta_{e}, g, \beta(s), b\right) l(b) k\left(g^{-1} h b\right) \kappa_{\beta(s), s}(d g) \gamma(b, d s) \lambda(d h) \nu_{\xi}^{*}(d b) .
$$

Applying the refined Campbell theorem (3.5) gives that $I$ equals

$$
\begin{aligned}
& \mathbb{E}_{\mathbb{P}} \iiint \int f\left(\theta_{h}^{-1}, g, \beta(s), \beta(t)\right) l(\beta(t)) k\left(g^{-1} h \beta(t)\right) \kappa_{\beta(s), s}(d g) \gamma\left(\theta_{h}^{-1}, \beta(t), d s\right) \kappa_{\beta(t), t}(d h) \xi(d t) \\
& =\mathbb{E}_{\mathbb{P}} \iiint \int f\left(\theta_{h}^{-1}, g, \beta(s), \beta(t)\right) l(\beta(t)) k\left(g^{-1} t\right) \kappa_{\beta(s), h^{-1} s}(d g) \gamma(h \beta(t), d s) \kappa_{\beta(t), t}(d h) \xi(d t) \\
& =\mathbb{E}_{\mathbb{P}} \iiint \int f\left(\theta_{h}^{-1}, g, \beta(s), \beta(t)\right) l(\beta(t)) k\left(g^{-1} t\right) \kappa_{\beta(s), h^{-1} s}(d g) \kappa_{\beta(t), t}(d h) \gamma(t, d s) \xi(d t),
\end{aligned}
$$

where we have the invariance property (4.1) of $\gamma$, invariance of $\beta$ and the fact that $\kappa_{\beta(t), t}$ is concentrated on $G_{\beta(t), t}$ (see Theorem 2.1 (ii)). By Theorem 2.1 (i) and (4.2)

$$
I=\mathbb{E}_{\mathbb{P}} \iiint \int f\left(\theta_{h}^{-1}, h^{-1} g, \beta(s), \beta(t)\right) l(\beta(t)) k\left(g^{-1} h t\right) \kappa_{\beta(t), t}(d h) \delta(s, d t) \kappa_{\beta(s), s}(d g) \eta(d s) .
$$

Using the invariance properties of the kernels $\delta$ and $\kappa$, we obtain that $I$ equals

$$
\begin{aligned}
\mathbb{E}_{\mathbb{P}} \iiint \int f\left(\theta_{h}^{-1} \circ \theta_{g}^{-1}, h^{-1}, \beta(s), \beta(t)\right) l(\beta(t)) \\
k(h g t) \kappa_{\beta(t), t}(d h) \delta\left(\theta_{g}^{-1}, \beta(s), d t\right) \kappa_{\beta(s), s}(d g) \eta(d s),
\end{aligned}
$$

where we have used that $\theta_{g h}^{-1}=\theta_{h}^{-1} \circ \theta_{g}^{-1}$ and that $g^{-1} s=\beta(s)$ for $s, g$ as in the above integral. At this stage we can use the refined Campbell theorem (3.5) for $\eta$ to obtain that

$$
I=\int \mathbb{E}_{Q_{\eta, b}} \iiint f\left(\theta_{h}^{-1}, h^{-1}, b, \beta(t)\right) l(\beta(t)) k(h g t) \kappa_{\beta(t), t}(d h) \delta(b, d t) \lambda(d g) \nu_{\eta}^{*}(d b) .
$$

Now take $h \in G$ and $t \in S$ with $h \beta(t)=t$. Then

$$
\int k(h g t) \lambda(d g)=\int k(g h \beta(t)) \lambda(d g)=\Delta\left(h^{-1}\right) \int k(g \beta(t)) \lambda(d g)=\Delta\left(h^{-1}\right) l(\beta(t))^{-1} .
$$

Therefore we obain from Fubini's theorem that $I$ equals the left-hand side of (4.3).

An immediate consequence of Theorem 4.1 is the following exchange formula for Palm pairs. A first version of this fundamental and very useful formula was obtained by Neveu (see e.g. [18]).

Corollary 4.2. Let $\xi$ and $\eta$ be an invariant random measures on $S$. Assume that $\xi$ and $\eta$ admit invariant Palm pairs $\left(\nu_{\xi}, Q_{\xi}\right)$ and $\left(\nu_{\eta}, Q_{\eta}\right)$, respectively. Then

$$
\begin{aligned}
\int & \mathbb{E}_{Q_{\eta, b}} \iint f\left(\theta_{g}^{-1}, g^{-1}, b, \beta(s)\right) \Delta^{*}(s) \kappa_{\beta(s), s}(d g) \xi(d s) \nu_{\eta}^{*}(d b) \\
& =\int \mathbb{E}_{Q_{\xi, b}} \iint f\left(\theta_{e}, g, \beta(s), b\right) \kappa_{\beta(s), s}(d g) \eta(d s) \nu_{\xi}^{*}(d b), \quad f \in(\mathcal{A} \otimes \mathcal{G} \otimes \mathcal{S} \otimes \mathcal{S})_{+} .
\end{aligned}
$$


Before discussing some examples we mention one consequence of Corollary 4.2 arising for a special choice of $f$.

Corollary 4.3. Under the hypothesis of Corollary 4.2 we have

$$
\begin{aligned}
\int \mathbb{E}_{Q_{\eta, b}} \iint f\left(\theta_{g}^{-1}, g^{-1} b, \beta(s)\right) \Delta^{*}(s) \kappa_{\beta(s), s}(d g) \xi(d s) \nu_{\eta}^{*}(d b) \\
=\int \mathbb{E}_{Q_{\xi, b}} \int f\left(\theta_{e}, s, b\right) \eta(d s) \nu_{\xi}^{*}(d b), \quad f \in(\mathcal{A} \otimes \mathcal{S} \otimes \mathcal{S})_{+} .
\end{aligned}
$$

Proof. Take $f \in(\mathcal{A} \otimes \mathcal{S} \otimes \mathcal{S})_{+}$and apply Corollary 4.2 with the function $\tilde{f}(\omega, g, s, t):=$ $f(\omega, g s, t)$.

Example 4.4. Assume that $S=G$ as in Example 2.3 and let $\xi$ and $\eta$ be invariant random measures on $G$. Then (4.4) means

$$
\mathbb{E}_{Q_{\eta}} \int f\left(\theta_{g}^{-1}, g^{-1}\right) \Delta\left(g^{-1}\right) \xi(d g)=\mathbb{E}_{Q_{\xi}} \int f\left(\theta_{e}, g\right) \eta(d g), \quad f \in(\mathcal{A} \otimes \mathcal{G})_{+},
$$

where $Q_{\xi}:=Q_{\xi, e}, Q_{\eta}:=Q_{\eta, e}$, cf. Example 3.3. For an Abelian group this is Neveu's exchange formula, see [18].

Example 4.5. Consider the quasi-transitive case of Example 3.4 and let $\xi, \eta, \gamma, \delta$ be as in Theorem 4.1 Then (4.3) easily implies for all $f \in(\mathcal{A} \otimes \mathcal{G})_{+}$and all $b, b^{\prime} \in O$ that

$$
\begin{aligned}
& \nu_{\eta}^{*}\{b\} \mathbb{E}_{Q_{\eta, b}} \iint f\left(\theta_{g}^{-1}, g^{-1}\right) \Delta^{*}(s) \kappa_{b^{\prime}, s}(d g) \mathbf{1}\left\{s \in G b^{\prime}\right\} \delta(b, d s) \\
&=\nu_{\xi}^{*}\left\{b^{\prime}\right\} \mathbb{E}_{Q_{\xi, b^{\prime}}} \iint f\left(\theta_{e}, g\right) \kappa_{b, s}(d g) \mathbf{1}\{s \in G b\} \gamma\left(b^{\prime}, d s\right) .
\end{aligned}
$$

The transitive special case of this result can be found in [12]. In case $G=S$ is an Abelian group we recover Theorem 3.6 in [13] (see also [11] for the non Abelian case).

\section{Characterization of Palm pairs}

We consider a kernel $\xi$ from $\Omega$ to $S$ that is uniformly $\sigma$-finite in the sense, that there is a partition $B_{1}, B_{2}, \ldots$ of $S$ such that $\xi\left(B_{i}\right)<\infty$ for any $i \in \mathbb{N}$. In contrast to the previous section we do not fix the underlying measure $\mathbb{P}$ on $(\Omega, \mathcal{A})$. Instead we fix a $\sigma$-finite measure $\nu$ on $S$ and a $\sigma$-finite kernel $Q$ from $S$ to $\Omega$ and ask for conditions that are necessary and sufficient for $(\nu, Q)$ to be a Palm pair of $\xi$ with respect to some $\sigma$-finite measure $\mathbb{P}$. If $\xi$ is invariant (see $(3.3))$ and the pair $(\nu, Q)$ is invariant (i.e. $\nu$ is invariant and $Q$ is invariant in the sense of (3.4)), then we want $\mathbb{P}$ to be invariant as well.

The following main result of this section is a significant extension of Mecke's [16] famous characterization of Palm measures of stationary random measures on an Abelian group. In the transitive special case of Example 3.3 the result has been derived in [19] and [12]. 
Theorem 5.1. Assume that $\xi$ and $(\nu, Q)$ are invariant. Then $(\nu, Q)$ is a Palm pair of $\xi$ with respect to some invariant $\sigma$-finite measure $\mathbb{P}$ on $(\Omega, \mathcal{A})$ iff $\nu \otimes Q$ is $\sigma$-finite,

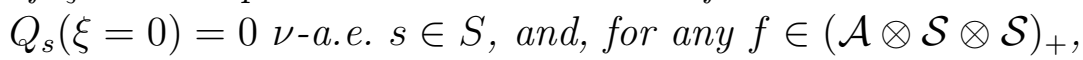

$$
\begin{aligned}
\int \mathbb{E}_{Q_{b}} \iint f\left(\theta_{g}^{-1}, g^{-1} b, \beta(s)\right) \Delta^{*}(s) \kappa_{\beta(s), s}(d g) \xi & (d s) \nu^{*}(d b) \\
& =\int \mathbb{E}_{Q_{b}} \int f\left(\theta_{e}, s, b\right) \xi(d s) \nu^{*}(d b) .
\end{aligned}
$$

For the proof of Theorem 5.1 it is convenient, to derive the result first without invariance assumptions, see Remark 3.2. This special case is of some independent interest.

Proposition 5.2. The pair $(\nu, Q)$ is a Palm pair of $\xi$ with respect to some $\sigma$-finite measure on $(\Omega, \mathcal{A})$ iff $\nu \otimes Q$ is $\sigma$-finite, $Q_{s}(\xi=0)=0$ for $\nu$-a.e. $s \in S$ and

$$
\iiint f(\omega, s, t) \xi(\omega, d t) Q_{s}(d \omega) \nu(d s)=\iiint f(\omega, t, s) \xi(\omega, d t) Q_{s}(d \omega) \nu(d s)
$$

for any measurable $f \in(\mathcal{A} \otimes \mathcal{S} \otimes \mathcal{S})_{+}$.

Proof. First, assume that $(\nu, Q)$ is a Palm pair of $\xi$ with respect to some $\sigma$-finite measure $\mathbb{P}$ on $\Omega$. This means

$$
\nu \otimes Q=C_{\xi}
$$

where $C_{\xi}$ is the Campbell measure of $\xi$ w.r.t. $\mathbb{P}$. As we have seen earlier, $C_{\xi}$ is $\sigma$-finite. Further

$$
\int Q_{s}(\xi=0) \nu(d s)=\mathbb{E}_{\mathbb{P}} \int \mathbf{1}\{\xi=0\} \xi(d s)=0,
$$

which implies $Q_{s}(\xi=0)=0$ for $\nu$-a.e. $s \in S$. To show (5.2) we take $f \in(\mathcal{A} \otimes \mathcal{S} \otimes \mathcal{S})_{+}$ and obtain from (5.3) and Fubini's theorem that the left hand-side of (5.2) equals

$$
\mathbb{E}_{\mathbb{P}} \iint f\left(\theta_{e}, s, t\right) \xi(d t) \xi(d s)=\mathbb{E}_{\mathbb{P}} \iint f\left(\theta_{e}, s, t\right) \xi(d s) \xi(d t) .
$$

Applying (5.3) again, we see that the latter expression coincides with the right-hand side of (5.2).

We now prove the converse implication. By $\sigma$-finiteness we may choose a measurable function $g^{\prime}>0$ on $\Omega \times S$ such that $(\nu \otimes Q) g^{\prime}<\infty$. Since $\xi$ is uniformly $\sigma$-finite, we may choose $\tilde{g}>0$ on $\Omega \times S$ with $0<\xi(\tilde{g})<\infty$ on $\{\xi \neq 0\}$. Now set $g:=g^{\prime} \wedge \tilde{g}$ and $h(\omega, s):=g(\omega, s) /(\xi g)(\omega)$, where $h(\omega, s):=0$ if $\xi(g)=0$. Define the measure $\mathbb{P}$ by

$$
\mathbb{P}(A):=\iint \mathbf{1}_{A}(\omega) h(\omega, s) Q_{s}(d \omega) \nu(d s), \quad A \in \mathcal{A} .
$$

By assumption on $Q$ we have $\mathbb{P}(\xi=0)=0$. The function $\xi(g)$ is finite and positive on $\{\xi \neq 0\}$. Furthermore,

$$
\mathbb{E}_{\mathbb{P}} \xi(g)=\iint(\xi g)(\omega) \frac{g(\omega, s)}{(\xi g)(\omega)} Q_{s}(d \omega) \nu(d s) \leq \iint g^{\prime}(\omega, s) Q_{s}(d \omega) \nu(d s)<\infty .
$$


Hence $\mathbb{P}$ is $\sigma$-finite. Moreover we have for $f \in(\mathcal{A} \otimes \mathcal{S})_{+}$

$$
\begin{aligned}
\mathbb{E}_{\mathbb{P}} \int f\left(\theta_{e}, t\right) \xi(d t) & =\iiint f(\omega, t) h(\omega, s) \xi(\omega, d t) Q_{s}(d \omega) \nu(d s) \\
& =\iiint f(\omega, s) h(\omega, t) \xi(\omega, d t) Q_{s}(d \omega) \nu(d s),
\end{aligned}
$$

where we have used (5.2) to get the second identity. This is just $(\nu \otimes Q) f$ since $\xi(h)=1$ on $\{\xi \neq 0\}$ by definition of $h$, and $Q_{s}(\xi=0)=0$ for $\nu$-a.e. $s \in S$.

If $(\nu, Q)$ is invariant, then the measure $\mathbb{P}$ defined by (5.4) turns out to be invariant. This leads to the following result.

Proposition 5.3. Assume that $\xi$ is invariant and that $(\nu, Q)$ is invariant. Then $(\nu, Q)$ is a Palm pair of $\xi$ with respect to some invariant $\sigma$-finite measure $\mathbb{P}$ on $(\Omega, \mathcal{A})$ iff $\nu \otimes Q$ is $\sigma$-finite, $Q_{s}(\xi=0)=0$ - -a.e. $s \in S$, and (5.2) holds for any measurable $f \in(\mathcal{A} \otimes \mathcal{S} \otimes \mathcal{S})_{+}$.

Proof. In view of Proposition 5.2 we only need to prove one implication. Therefore we assume (5.2) and the other two conditions on $(\nu, Q)$. We have to show that the measure $\mathbb{P}$ defined in (5.4) is invariant for invariant $\nu$ and $Q$. Take $f \in \mathcal{A}_{+}$and $g \in G$. By invariance of $Q$ and invariance of $\nu$,

$$
\begin{aligned}
\mathbb{E}_{\mathbb{P}} f \circ \theta_{g} & =\iint f\left(\theta_{g} \omega\right) h(\omega, s) Q_{s}(d \omega) \nu(d s) \\
& =\iint f(\omega) h\left(\theta_{g}^{-1} \omega, s\right) Q_{g s}(d \omega) \nu(d s) \\
& =\iint f(\omega) h\left(\theta_{g}^{-1} \omega, g^{-1} s\right) Q_{s}(d \omega) \nu(d s) .
\end{aligned}
$$

Since $(\nu, Q)$ is a Palm pair of $\xi($ w.r.t. $\mathbb{P})$ we obtain

$$
\begin{aligned}
\mathbb{E}_{\mathbb{P}} f \circ \theta_{g} & =\mathbb{E}_{\mathbb{P}} \int f\left(\theta_{e}\right) h\left(\theta_{g}^{-1}, g^{-1} s\right) \xi(d s) \\
& =\iint f(\omega) h\left(\theta_{g}^{-1} \omega, s\right) \xi\left(\theta_{g}^{-1} \omega, d s\right) \mathbb{P}(d \omega)=\mathbb{E}_{\mathbb{P}} f,
\end{aligned}
$$

where we have used in the last step that $\int h\left(\theta_{g}^{-1} \omega, s\right) \xi\left(\theta_{g}^{-1} \omega, d s\right)=1$ for $\mathbb{P}$-a.e. $\omega$ since $\{\xi \neq 0\}$ is invariant and has a complement of measure 0 .

Proof of Theorem 5.1. If $(\nu, Q)$ is a Palm pair of $\xi$ then $\sigma$-finiteness of $\nu \otimes Q$ and $Q_{s}(\xi=0)=0$ for $\nu$-a.e. $s \in S$, have been shown in Proposition 5.2. Equation (5.1) is a special case of (4.5).

Conversely assume the regularity conditions and (5.1). By Proposition 5.3 it is enough to show that this implies (5.2). By means of (2.13) we have

$$
\begin{aligned}
\iiint f(\omega, s, t) \xi(\omega, d t) Q_{s}(d \omega) \nu(d s) & =\iint \mathbb{E}_{Q_{s}} \int f\left(\theta_{e}, s, t\right) \xi(d t) \mu_{b}(d s) \nu^{*}(d b) \\
& =\iint \mathbb{E}_{Q_{h b}} \int f\left(\theta_{e}, h b, t\right) \xi(d t) \lambda(d h) \nu^{*}(d b) \\
& =\iint \mathbb{E}_{Q_{b}} \int f\left(\theta_{h}, h b, h t\right) \xi(d t) \lambda(d h) \nu^{*}(d b),
\end{aligned}
$$


where we used invariance of $Q$ and $\xi$ in the last step. Using the stochastic kernel $\kappa$ this last expression can be written as

$$
\int \mathbb{E}_{Q_{b}} \iiint f\left(\theta_{h}, h b, h g \beta(t)\right) \kappa_{\beta(t), t}(d g) \xi(d t) \lambda(d h) \nu^{*}(d b),
$$

and this equals

$$
\int \mathbb{E}_{Q_{b}} \iiint f\left(\theta_{h g^{-1}}, h g^{-1} b, h \beta(t)\right) \lambda(d h) \Delta\left(g^{-1}\right) \kappa_{\beta(t), t}(d g) \xi(d t) \nu^{*}(d b),
$$

by Fubini's theorem and a characteristic property of the modular function. Now apply (5.1) to the function $(\omega, s, t) \mapsto \int f\left(\theta_{h} \omega, h s, h t\right) \lambda(d h)$ to write this as

$$
\int \mathbb{E}_{Q_{b}} \iint f\left(\theta_{h}, h t, h b\right) \lambda(d h) \xi(d t) \nu^{*}(d b) .
$$

By Fubini's theorem and invariance of $Q$ and $\xi$ this can be written as

$$
\begin{aligned}
\iint \mathbb{E}_{Q_{h b}} \int f\left(\theta_{e}, t, h b\right) \xi(d t) \lambda(d h) \nu^{*}(d b) & =\iint \mathbb{E}_{Q_{s}} \int f\left(\theta_{e}, t, s\right) \xi(d t) \mu_{b}(d s) \nu^{*}(d b) \\
& =\iiint f(\omega, t, s) \xi(\omega, d t) Q_{s}(d \omega) \nu(d s),
\end{aligned}
$$

where we have used (2.13) for the second equality.

\section{The mass-transport principle}

In this section we will show that Theorem 4.1 contains a mass-conservation law. Recall our basic properness assumption for the operation of $G$ on $S$. Let $M$ denote a $\sigma$-finite invariant measure on $S \times S$, which is given the interpretation that $M(C \times D)$ represents mass being transported out of $C$ into $D$. Then the main result of Subsection 6.1 says that on any set $B$ with a symmetry property with respect to the operating group $G$ the mass transported out of $B$ - suitably weighted in the non-unimodular case - equals the total mass transported into $B$. For a precise formulation of this mass-transport principle (short: $M T P$ ) fix $k>0$ as in $(2.3)$ and define a measurable function $\tilde{\Delta}: S \times S \rightarrow(0, \infty)$ by

$$
\tilde{\Delta}(s, t):=\frac{\mu_{t} k}{\mu_{\beta(t)} k} \frac{\mu_{\beta(s)} k}{\mu_{s} k} .
$$

Note that $\tilde{\Delta}(s, g s)=\Delta\left(g^{-1}\right), g \in G, s \in S$. A symmetric set is a set $B \in \mathcal{S}$ satisfying $0<\mu_{b}(B)=\mu_{b^{\prime}}(B)<\infty, b, b^{\prime} \in O$. The following result will be obtained as a special case of Theorem 6.8 in Subsection 6.1.

Theorem 6.1. For any $\sigma$-finite and jointly invariant measure $M$ on $S \times S$ and any symmetric $B \in \mathcal{S}$ we have

$$
\int \tilde{\Delta}(s, t) \mathbf{1}_{B}(s) M(d(s, t))=\int \mathbf{1}_{B}(t) M(d(s, t)) .
$$


In the transitive case, any set $B \in \mathcal{S}$ with positive and finite invariant measure is symmetric and Theorem 6.1 can be simplified as follows.

Corollary 6.2. If $G$ operates transitively on $S$ then for any $\sigma$-finite invariant measure $M$ on $S \times S$ and any $B \in \mathcal{S}$ we have

$$
\int \tilde{\Delta}(s, t) \mathbf{1}_{B}(s) M(d(s, t))=\int \mathbf{1}_{B}(t) M(d(s, t)),
$$

where $\tilde{\Delta}(s, t)=\Delta\left(g^{-1}\right)$ for any $g \in G$ with $g s=t$.

Up to an integrability issue Theorem 6.1 implies the following stochastic analogue for invariant random measures. In Subsection 6.2 we shall derive this result from the transport formula of Theorem 4.1. A function on $\Omega \times S$ (or other product spaces) is invariant if it is invariant under joint shifts of the arguments.

Theorem 6.3. Let $\xi, \eta$ be invariant random measures on $S$, and $\gamma, \delta$ invariant kernels from $\Omega \times S$ to $S$ such that for $\mathbb{P}$-a.e. $\omega$

$$
\iint 1\{(s, t) \in \cdot\} \gamma(\omega, s, d t) \xi(\omega, d s)=\iint 1\{(s, t) \in \cdot\} \delta(\omega, t, d s) \eta(\omega, d t) .
$$

Then for a symmetric set $B \in \mathcal{S}$ and any invariant $h \in(\mathcal{A} \otimes \mathcal{S} \otimes \mathcal{S})_{+}$we have

$$
\mathbb{E} \iint \tilde{\Delta}(s, t) \mathbf{1}_{B}(s) h\left(\theta_{e}, s, t\right) \gamma(s, d t) \xi(d s)=\mathbb{E} \iint \mathbf{1}_{B}(t) h\left(\theta_{e}, s, t\right) \delta(t, d s) \eta(d t) .
$$

\subsection{Deterministic transport principle}

Imagine there is some mass distributed over the space $S$ and that we transport from each $s \in S$ to each $t \in S$ some mass $m(s, t)$ in an invariant way, i.e. we assume that

$$
m(g s, g t)=m(s, t), \quad g \in G .
$$

One might guess that the total mass being transported from one orbit in $S$ to some fixed point in $S$ does only depend on the orbit of this fixed point and in fact equals the total mass being transported from any representative of the initial orbit into the whole orbit of our target point.

To make this precise fix some representatives $b, b^{\prime} \in O$ and consider their corresponding orbital invariant measures $\mu_{b}, \mu_{b^{\prime}}$ which are $\sigma$-finite by our properness assumption. Recall the definition $(2.10)$ of the function $\Delta^{*}$. The calculation

$$
\begin{aligned}
\int m(b, s) \Delta^{*}(s) \mu_{b^{\prime}}(d s) & =\int m\left(b, g b^{\prime}\right) \Delta\left(g^{-1}\right) \lambda(d g)=\int m\left(b, g^{-1} b^{\prime}\right) \lambda(d g) \\
& =\int m\left(g b, b^{\prime}\right) \lambda(d g)=\int m\left(s, b^{\prime}\right) \mu_{b}(d s)
\end{aligned}
$$

yields a basic balance equation between any two orbits: 
Corollary 6.4. Let $m \in(\mathcal{S} \otimes \mathcal{S})_{+}$be invariant. Then

$$
\int m(b, s) \Delta^{*}(s) \mu_{b^{\prime}}(d s)=\int m\left(s, b^{\prime}\right) \mu_{b}(d s), \quad b, b^{\prime} \in O .
$$

Corollary 6.4 is in fact a consequence of (4.4). To see this specialize $\xi$ and $\eta$ to $\xi:=\mu_{b}, \eta:=\mu_{b^{\prime}}$, choose respective supporting measures $\nu_{\mu_{b}}:=\mu_{b}$ and $\nu_{\mu_{b^{\prime}}}:=\mu_{b^{\prime}}$, note that $Q_{\xi, b}=\mathbb{P}=Q_{\eta, b}, b \in O$, and that $\mu_{b}^{*}=\delta_{b}$, replace $f(\omega, g, s, t):=g(\omega) m(s, t)$ where $g$ is chosen such that $\mathbb{E}_{\mathbb{P}}[g]=1$, factor out the $\sigma$-finite and invariant $\mathbb{P}$ on both sides and finally use invariance of $m$.

Corollary 6.4 leads to the following mass-transport principle on any system $O$ of orbit representatives. Given two invariant measures $\mu$ and $\nu$ on $S$ we may interpret $\mu$ as mass distributed within $S$ while $\nu$ on the other hand can be thought of holes where mass can be stored. Consider invariant kernels $\gamma$ and $\delta$ on $S$, where an application of $\gamma$ to $\mu$ may be thought of resizing and redistributing the mass $\mu$, while an application of $\delta$ to $\nu$ may be interpreted as streching or shrinking and relocating the holes $\nu$. Therefore one might call $\gamma$ and $\delta$ weighted transport kernels, see [13].

Corollary 6.5. Let $\mu, \nu$ be $\sigma$-finite invariant measures on $S$ and $\gamma, \delta$ invariant kernels on $S$ satisfying

$$
\iint 1\{(s, t) \in \cdot\} \gamma(s, d t) \mu(d s)=\iint 1\{(s, t) \in \cdot\} \delta(t, d s) \nu(d t) .
$$

Then for any invariant $m \in(\mathcal{S} \otimes \mathcal{S})_{+}$

$$
\iint m(b, t) \Delta^{*}(t) \gamma(b, d t) \mu^{*}(d b)=\iint m(s, b) \delta(b, d s) \nu^{*}(d b) .
$$

Proof. Specializing $\xi:=\mu, \eta:=\nu$ in Theorem 4.1 and following similar steps as above yields the result.

The following version of the above deterministic MTP has been established in [2] in the case of finitely many orbits (also see [14], Chapter 8).

Corollary 6.6. Let $G$ operate properly on the countable set $S$. Then

$$
\sum_{b, b^{\prime} \in O} \sum_{s \in G b^{\prime}} m(b, s) \lambda\left(G_{s, s}\right)=\sum_{b, b^{\prime} \in O} \sum_{s \in G b^{\prime}} \lambda\left(G_{b^{\prime}, b^{\prime}}\right) m(s, b)
$$

and if $G$ is unimodular then

$$
\sum_{b \in O} \frac{1}{\lambda\left(G_{b, b}\right)} \sum_{s \in S} m(b, s)=\sum_{b \in O} \frac{1}{\lambda\left(G_{b, b}\right)} \sum_{s \in S} m(s, b) .
$$

Proof. Putting $\mu=\nu:=\sum_{b \in O} \mu_{b}$ (hence $\mu^{*}=\nu^{*}=\sum_{b \in O} \delta_{b}$ ) in (6.5) yields

$$
\sum_{b, b^{\prime} \in O} \lambda\left(G_{b^{\prime}, b^{\prime}}\right) \sum_{s \in G b^{\prime}} m(b, s) \Delta^{*}(s)=\sum_{b, b^{\prime} \in O} \lambda\left(G_{b^{\prime}, b^{\prime}}\right) \sum_{s \in G b^{\prime}} m(s, b)
$$


for any measurable invariant $m$. Using (2.11) this simplifies to

$$
\sum_{b, b^{\prime} \in O} \sum_{s \in G b^{\prime}} m(b, s) \lambda\left(G_{s, s}\right)=\sum_{b, b^{\prime} \in O} \sum_{s \in G b^{\prime}} \lambda\left(G_{b^{\prime}, b^{\prime}}\right) m(s, b) .
$$

If $G$ is unimodular then $\lambda\left(G_{b^{\prime}, b^{\prime}}\right)$ on the right may be replaced by $\lambda\left(G_{s, s}\right)$ and we may also replace $m$ by the $G$-invariant function $m(t, s) \frac{1}{\lambda\left(G_{t, t}\right) \lambda\left(G_{s, s}\right)}$ which then yields the assertion.

We seek for an appropriate formulation of Corollary 6.5 without use of a fixed system of representatives of the orbits. For this choose any $v \in \mathcal{S}_{+}$with the property that $0<\mu_{s} v<\infty$ for each $s \in S$ and define similarly as in (6.1)

$$
\Delta_{v}(s, t):=\frac{\mu_{t} v}{\mu_{s} v}, \quad s, t \in G .
$$

By means of another function $w \in \mathcal{S}_{+}$with $0<\mu_{s} w<\infty$ for any $s \in S$ being compatible with $v$ in the sense that

$$
\frac{\mu_{b} w}{\mu_{b} v}=\frac{\mu_{b^{\prime}} w}{\mu_{b^{\prime}} v}, \quad b, b^{\prime} \in O
$$

we may, since $\Delta^{*}(s)=\mu_{s}(v) / \mu_{\beta(s)}(v)$, express $\Delta^{*}$ as

$$
\Delta^{*}(s)=\Delta^{*}(s) \frac{\mu_{\beta(s)}(v)}{\mu_{\beta(s)}(w)} \frac{\mu_{b}(w)}{\mu_{b}(v)}=\Delta_{v}(b, s) \frac{\mu_{b}(w)}{\mu_{\beta(s)}(w)}, \quad b \in O, s \in S
$$

where we have used assumption (6.7).

Proposition 6.7. Let $\mu, \nu$ be $\sigma$-finite invariant measures on $S$ and $\gamma$ and $\delta$ be invariant kernels on $S$ satisfying

$$
\iint \mathbf{1}\{(s, t) \in \cdot\} \gamma(s, d t) \mu(d s)=\iint \mathbf{1}\{(s, t) \in \cdot\} \delta(t, d s) \nu(d t) .
$$

Let $v, w \in \mathcal{S}_{+}$be as in $(6.7)$ and $m \in(\mathcal{S} \otimes \mathcal{S})_{+}$be invariant. Then

$$
\iint \Delta_{v}(s, t) w(s) m(s, t) \gamma(s, d t) \mu(d s)=\iint w(t) m(s, t) \delta(t, d s) \nu(d t) .
$$

Proof. Replacing $\Delta^{*}(t)$ in (6.5) by the left-hand side of (6.8), we get

$$
\iint m(b, t) \Delta_{v}(b, t) \frac{\mu_{b}(w)}{\mu_{\beta(t)}(w)} \gamma(b, d t) \mu^{*}(d b)=\iint m(s, b) \delta(b, d s) \nu^{*}(d b) .
$$

Applying this with $m$ replaced by the invariant function $m(s, t) \mu_{\beta(t)}(w)$ yields

$$
\iint m(b, t) \Delta_{v}(b, t) \mu_{b}(w) \gamma(b, d t) \mu^{*}(d b)=\iint m(s, b) \mu_{b}(w) \delta(b, d s) \nu^{*}(d b) .
$$

Fubini's theorem yields

$$
\iiint m(b, t) \Delta_{v}(b, t) w(s) \gamma(b, d t) \mu_{b}(d s) \mu^{*}(d b)=\iiint m(s, b) w(t) \delta(b, d s) \mu_{b}(d t) \nu^{*}(d b) .
$$


By invariance of $m \Delta_{v}$ and invariance of $\gamma$ the integral $\int m(s, t) \Delta_{v}(s, t) \gamma(s, d t)$ does not depend on $s \in G b$ for fixed $b \in O$. A similar remark applies to the inner integral of the above right-hand side. Applying (2.13) we obtain the asserted identity.

Using a result of Kallenberg in [10], Proposition 6.7 can be formulated equivalently in a shorter way.

Theorem 6.8. Let $v, w \in \mathcal{S}_{+}$satisfy (6.7). Then for any jointly invariant $\sigma$-finite measure $M$ on $S \times S$ we have

$$
\int \Delta_{v}(s, t) w(s) M(d(s, t))=\int w(t) M(d(s, t)) .
$$

Proof. According to Kallenberg [10] Corollary 3.6, there exist invariant disintegrations of the form

$$
M(d(s, t))=\gamma(s, d t) \mu(d s), \quad M(d(s, t))=\gamma(t, d s) \nu(d t),
$$

where $\mu, \nu$ are invariant $\sigma$-finite measures on $S$ and $\gamma, \delta$ are invariant kernels on $S$. Then (6.9) holds, and Theorem 6.7 implies the assertion.

Remark 6.9. To recover the more intuitive Theorem 6.1 from the last theorem just note that $v:=k(s) / \mu_{\beta(s)} k$ and $w:=\mathbf{1}_{B}$ for a symmetric $B \in \mathcal{S}$ is an admissable choice since

$$
\frac{\mu_{b} v}{\mu_{b}(B)}=\frac{1}{\mu_{b}(B)}=\frac{1}{\mu_{b^{\prime}}(B)}=\frac{\mu_{b^{\prime}} v}{\mu_{b^{\prime}}(B)}, \quad b, b^{\prime} \in O .
$$

\subsection{Transport principle for stationary random measures}

In this section we show how Theorem 6.3 follows from the transport formula in Theorem 4.1. To this end we begin with a modification of the refined Campbell theorem for invariant functions.

Lemma 6.10. Let $\xi$ be a invariant random measure on $S$ and $\left(\nu_{\xi}, Q_{\xi}\right)$ an invariant Palm pair of $\xi$. Then for any $G$-invariant $f \in(\mathcal{A} \otimes \mathcal{S})_{+}$and any $v \in \mathcal{S}_{+}$

$$
\int \mathbb{E}_{Q_{\xi, b}}\left[f\left(\theta_{e}, b\right)\right] \mu_{b}(v) \nu_{\xi}^{*}(d b)=\mathbb{E}_{\mathbb{P}} \int f\left(\theta_{e}, s\right) v(s) \xi(d s) .
$$

Proof. The left side equals

$$
\int \mathbb{E}_{Q_{\xi, b}} \int f\left(\theta_{e}, b\right) v(g b) \lambda(d g) \nu_{\xi}^{*}(d b)=\mathbb{E} \iint f\left(\theta_{g}^{-1}, \beta(s)\right) v(g \beta(s)) \kappa_{\beta(s), s}(d g) \xi(d s),
$$

where we used the refined Campbell theorem 3.1. Invariance of $f$ yields the result.

Applying this modified Campbell formula for invariant functions to the transport formula of Theorem 4.1 yields Theorem 6.3: 
Proof of Theorem 6.3. Replacing $f(\omega, g, s, t)$ in $(4.3)$ by $h(\omega, g s, t)$ for $h \in(\mathcal{A} \otimes \mathcal{S} \otimes \mathcal{S})_{+}$ yields

$$
\begin{aligned}
& \int \mathbb{E}_{Q_{\eta, b}} \iint h\left(\theta_{g}^{-1}, g^{-1} b, \beta(s)\right) \Delta^{*}(s) \kappa_{\beta(s), s}(d g) \delta(b, d s) \nu_{\eta}^{*}(d b) \\
&=\int \mathbb{E}_{Q_{\xi, b}} \iint h\left(\theta_{e}, g \beta(s), b\right) \kappa_{\beta(s), s}(d g) \gamma(b, d s) \nu_{\xi}^{*}(d b) .
\end{aligned}
$$

If $h$ is invariant this reduces to

$$
\int \mathbb{E}_{Q_{\eta, b}} \int h\left(\theta_{e}, b, s\right) \Delta^{*}(s) \delta(b, d s) \nu_{\eta}^{*}(d b)=\int \mathbb{E}_{Q_{\xi, b}} \int h\left(\theta_{e}, s, b\right) \gamma(b, d s) \nu_{\xi}^{*}(d b) .
$$

Substituting $\Delta^{*}(s)$ via $(6.8)$ and replacing $h(\omega, t, s)$ by $h(\omega, t, s) \mu_{\beta(s)}(w)$ yields for any invariant $h$

$$
\int \mathbb{E}_{Q_{\eta, b}} \int h\left(\theta_{e}, b, s\right) \Delta_{v}(b, s) \mu_{b}(w) \delta(b, d s) \nu_{\eta}^{*}(d b)=\int \mathbb{E}_{Q_{\xi, b}} \int h\left(\theta_{e}, t, b\right) \mu_{b}(w) \gamma(b, d t) \nu_{\xi}^{*}(d b) .
$$

Here $(\omega, t) \mapsto \int h(\omega, t, s) \Delta_{v}(t, s) \delta(\omega, t, d s)$ and $(\omega, s) \mapsto \int h(\omega, t, s) \gamma(\omega, s, d t)$ are clearly invariant by invariance of the transports $\gamma, \delta$ and the function $h$. Thus (6.11) may be applied to both sides of the last equation to yield

$$
\mathbb{E} \iint h\left(\theta_{e}, t, s\right) \Delta_{v}(t, s) w(t) \delta(t, d s) \eta(d t)=\mathbb{E} \iint h\left(\theta_{e}, t, s\right) w(s) \gamma(s, d t) \xi(d s) .
$$

Since $\Delta_{v}(s, t)=\Delta_{v}(t, s)^{-1}$ this is clearly equivalent to saying that for any invariant $h$

$$
\mathbb{E} \iint h\left(\theta_{e}, t, s\right) w(t) \delta(t, d s) \eta(d t)=\mathbb{E} \iint h\left(\theta_{e}, t, s\right) \Delta_{v}(s, t) w(s) \gamma(s, d t) \xi(d s) .
$$

Choosing $v, w$ as in Remark 6.9 yields the result.

A useful corollary of Theorem 6.3 is to specialize $\gamma(\omega, s, d t):=m(\omega, s, t) \eta(\omega, d t)$ and $\delta(\omega, t, d s):=m(\omega, s, t) \xi(\omega, d s)$ for any invariant $m \in(\mathcal{A} \otimes \mathcal{S} \otimes \mathcal{S})_{+}:$

Corollary 6.11. For invariant random measures $\xi, \eta$ and invariant $m \in(\mathcal{A} \otimes \mathcal{S} \otimes \mathcal{S})_{+}$ we have

$$
\mathbb{E} \iint \Delta_{v}(s, t) w(s) m\left(\theta_{e}, s, t\right) \xi(d s) \eta(d t)=\mathbb{E} \iint w(t) m\left(\theta_{e}, s, t\right) \xi(d s) \eta(d t) .
$$

\section{Appendix}

If $G$ operates measurably on $S$ and $T$ then following Kallenberg in [10] we call a measure on a product space $S \times T$ jointly $G$-invariant if it is invariant with respect to the diagonal operation

$$
\theta_{g}(s, t):=(g s, g t), \quad g \in G, s \in S, t \in T .
$$

We call a kernel $\kappa$ from $S$ to $T$ measurably $\sigma$-finite if for each $s \in S$ there is a measurable partition $B_{1}^{s}, B_{2}^{s}, \ldots$ of $T$ such that $(s, t) \mapsto 1_{B_{i}^{s}}(t)$ is measurable for all $i \in \mathbb{N}$ and $\kappa\left(s, B_{i}^{s}\right)<\infty, s \in S$. It is easy to prove that a kernel from $S$ to $T$ is measurably 
$\sigma$-finite if and only if there exists a measurable function $f>0$ on $S \times T$ such that $\kappa_{s} f_{s}<\infty, s \in S$, where $f_{s}:=f(s, \cdot)$.

Our aim is to disintegrate measurably labeled families of jointly $G$-invariant measures $\left\{M_{r}\right\}_{r \in R}$ on a certain product space in an invariant and measurable way. For this we need the following two lemmas which are straightforward adaptions of standard arguments concerning the existence of disintegrations of measures on product spaces (see e.g. [9] Theorem 6.3) and their respective $G$-invariant versions for jointly $G$-invariant measures found by Kallenberg in [10].

Lemma 7.1. Let $R, S, T$ be measurable spaces where $S$ and $T$ are Borel, $M$ be a measurably $\sigma$-finite kernel from $R$ to $S \times T$. Then there is a stochastic kernel $\nu$ from $R$ to $S$ and a measurably $\sigma$-finite kernel $\kappa$ from $R \times S$ to $T$ such that

$$
M_{r}=\nu_{r} \otimes \kappa_{r}, \quad r \in R .
$$

In addition if $\nu^{\prime}$ is a given measurably $\sigma$-finite kernel from $R$ to $S$ such that $M_{r}(\cdot \times T) \ll$ $\nu_{r}^{\prime}, r \in R$, there is a suitable kernel $\kappa^{\prime}$ from $R \times S$ to $T$ such that

$$
M_{r}=\nu_{r}^{\prime} \otimes \kappa_{r}^{\prime}, \quad r \in R .
$$

Proof. Without loss of generality we may assume that $M_{r}(S \times T)>0, r \in R$. Since $M$ is measurably $\sigma$-finite we may choose a measurable function $f>0$ on $R \times S \times T$ such that $M_{r} f_{r}=1, r \in R$, and define the stochastic kernel $P$ from $R$ to $S \times T$ as $P_{r}:=f_{r} \cdot M_{r}$, $r \in R$. Then $\nu_{r}:=P_{r}(\cdot \times T)$ is a stochastic kernel from $R$ to $S$. Since $T$ is Borel we may assume $T \in \mathcal{B}(\mathbb{R})$ and since $S$ is Borel (in particular its $\sigma$-algebra is seperable) there exists according to Dellacherie/Meyer V.58 for each $q \in \mathbb{Q}$ an $\mathcal{R} \otimes \mathcal{S}$-measurable function $m(r, s, q)$ such that

$$
m(r, s, q)=\frac{d P_{r}(\cdot \times(-\infty, q])}{d \nu_{r}}(s), \quad \nu_{r} \text {-a.e. } s \in S, r \in R .
$$

For $r \in R$ the sets

$$
A_{r}:=\left\{s \in S: m(r, s, \cdot) \text { is monotone on } \mathbb{Q}, \lim _{q \rightarrow \infty} m(r, s, q)=1, \lim _{q \rightarrow-\infty} m(r, s, q)=0\right\}
$$

are defined by countably many measurable conditions, hence $A_{r} \in \mathcal{S}$ and in addition $(r, s) \rightarrow \mathbf{1}_{A_{r}}(s)$ is clearly measurable. Since each of the defining conditions holds for $\nu_{r}$-a.e. $s \in S$ we also have $\nu_{r}\left(A_{r}\right)=1, r \in R$. Now define

$$
\bar{m}(r, s, x):=\mathbf{1}_{A_{r}}(s) \inf _{q>x} m(r, s, q)+\mathbf{1}_{A_{r}^{c}}(s) 1\{x \geq 0\}, \quad x \in \mathbb{R}, s \in S, r \in R .
$$

Then $\bar{m}$ is measurable in $(r, s)$ and every $\bar{m}(r, s, \cdot)$ is a distribution function. By Proposition 2.14 in [9] there are probability measures $Q(r, s, \cdot)$ on $\mathbb{R}$ such that

$$
Q(r, s,(-\infty, x])=\bar{m}(r, s, x), \quad x \in \mathbb{R} .
$$

An easy monotone class argument now shows that even $Q(r, s, B)$ is measurable in $(r, s)$ for any $B \in \mathcal{B}(\mathbb{R})$, hence $Q$ is a kernel from $R \times S$ to $T$. Equation (7.1) implies

$$
\int f(s) P_{r}(d s \times(-\infty, q])=\int f(s) Q(r, s,(-\infty, q]) \nu_{r}(d s), \quad f \in \mathcal{S}_{+}, q \in \mathbb{Q}, r \in R
$$


and by monotone convergence this extends to

$$
\int f(s) P_{r}(d s \times(-\infty, x))=\int f(s) Q(r, s,(-\infty, x)) \nu_{r}(d s), \quad f \in \mathcal{S}_{+}, x \in \mathbb{R}, r \in R .
$$

Another monotone class argument yields

$$
\int f(s) P_{r}(d s \times B)=\int f(s) Q(r, s, B) \nu_{r}(d s), \quad f \in \mathcal{S}_{+}, B \in \mathcal{B}(\mathbb{R}), r \in R .
$$

In particular $Q\left(r, s, T^{c}\right)=0, \nu_{r}$-a.e.s $\in S, r \in R$. Now define

$$
\tilde{\kappa}(r, s, \cdot):=Q(r, s, \cdot) \mathbf{1}\{Q(r, s, T)=1\}+\delta_{t} \mathbf{1}\{Q(r, s, T)<1\}, \quad r \in R, s \in S,
$$

for some fixed $t \in T$. Then $\tilde{\kappa}$ is a stochastic kernel from $R \times S$ to $T$ such that

$$
P_{r}=\nu_{r} \otimes \tilde{\kappa}_{r}, \quad r \in R .
$$

This is clearly equivalent to

$$
M_{r}=\nu_{r} \otimes \kappa_{r}, \quad r \in R,
$$

where $\kappa(r, s, A):=\int \frac{1}{f(r, s, t)} \mathbf{1}_{A}(t) \tilde{\kappa}(r, s, d t), A \in \mathcal{T}$, and thus proves the first assertion.

If $\nu^{\prime}$ is a given kernel from $R$ to $S$ with the property $M_{r}(\cdot \times T) \ll \nu_{r}^{\prime}, r \in R$, then $\nu_{r} \sim M_{r}(\cdot \times T) \ll \nu_{r}^{\prime}, r \in R$, and again by Dellacherie/Meyer V.58 we may choose a measurable function $f: R \times S \rightarrow[0, \infty]$ such that

$$
f(r, s)=\frac{d \nu_{r}}{d \nu_{r}^{\prime}}(s), \quad \nu_{r}^{\prime} \text {-a.e. } s \in S .
$$

Then

$$
M_{r}=\nu_{r}^{\prime} \otimes \kappa_{r}^{\prime}, \quad r \in R,
$$

where $\kappa^{\prime}(r, s, \cdot):=f(r, s) \kappa(r, s, \cdot)$ and which proves the second statement.

Now Kallenbergs perfection technique may be applied to derive a suitably invariant version of $\kappa$ in a way that preserves measurability in $r$ :

Lemma 7.2. Let $R, S, T$ be measurable spaces where $S$ and $T$ are Borel and let $G$ operate measurably on both $S$ and $T$. Let $M$ be a measurably $\sigma$-finite kernel from $R$ to $S \times T$ such that $M_{r}$ is jointly $G$-invariant for each $r \in R$ and $\nu$ a measurably $\sigma$-finite kernel from $R$ to $S$ such that $\nu_{r}$ is a $G$-invariant measure on $S$ with $M_{r}(\cdot \times T) \ll \nu_{r}$ for $r \in R$. Then there is a kernel $\kappa$ from $R \times S$ to $T$ with the covariance property

$$
\kappa_{r}(g s, A)=\kappa_{r}\left(s, \theta_{g}^{-1} A\right), \quad A \in \mathcal{T}, s \in S, g \in G, r \in R,
$$

such that

$$
M_{r}=\nu_{r} \otimes \kappa_{r}, \quad r \in R .
$$

Proof. Lemma 7.1 yields a kernel $\kappa$ from $R \times S$ to $T$ disintegrating the kernel $M$ measurably, i.e. $M_{r}=\nu_{r} \otimes \kappa_{r}, r \in R$. Invariance of $M_{r}$ and $\nu_{r}$ implies for any $f \in(\mathcal{S} \otimes \mathcal{T})_{+}$ that

$$
\iint f(s, t) \kappa_{r, g s}(d t) \nu_{r}(d s)=\iint f(s, t) \kappa_{r, s} \circ \theta_{g}^{-1}(d t) \nu_{r}(d s), \quad g \in G, r \in R .
$$


Since $T$ is Borel this gives

$$
\kappa_{r, g s}=\kappa_{r, s} \circ \theta_{g}^{-1}, \quad \nu_{r} \text {-a.e. } s \in S, g \in G, r \in R \text {. }
$$

Fix some right Haar measure $\tilde{\lambda}$ on $G$. Fubinis theorem yields in particular

$$
\kappa_{r, g s}=\kappa_{r, s} \circ \theta_{g}^{-1}, \quad \tilde{\lambda} \text {-a.e. } g \in G, \nu_{r} \text {-a.e. } s \in S, r \in R \text {. }
$$

Let $l \geq 0$ be some measurable function on $G$ with $\tilde{\lambda} l=1$ and set

$$
\bar{\kappa}_{r, s}:=\int\left(\kappa_{r, h s} \circ \theta_{h}\right)(l \cdot \tilde{\lambda})(d h) .
$$

(7.2) implies $\bar{\kappa}_{r, s}=\kappa_{r, s}, \nu_{r}$-a.e. $s \in S, r \in R$. The following calculation yields for any $f \in \mathcal{T}_{+}, h \in G, s \in S$ and $r \in R$

$$
\begin{aligned}
\left|\left(\bar{\kappa}_{r, s}-\bar{\kappa}_{r, h s} \circ \theta_{h}\right) f\right| & =\left|\iint\left(\kappa_{r, p s} \circ \theta_{p}-\kappa_{r, q h s} \circ \theta_{q h}\right)(f)(l \cdot \tilde{\lambda})^{2}(d(p, q))\right| \\
& \leq \iint l(p) l\left(q h^{-1}\right)\left|\left(\kappa_{r, p s} \circ \theta_{p}-\kappa_{r, q s} \circ \theta_{q}\right)(f) \tilde{\lambda}^{2}(d(p, q))\right| .
\end{aligned}
$$

Setting

$$
A_{r}:=\left\{s \in S: \kappa_{r, p s} \circ \theta_{p}=\kappa_{r, q s} \circ \theta_{q}, \tilde{\lambda}^{2} \text {-a.e. }(p, q) \in G^{2}\right\}, \quad r \in R,
$$

this implies that

$$
\bar{\kappa}_{r, s}=\bar{\kappa}_{r, h s} \circ \theta_{h}, \quad h \in G, s \in A_{r}, r \in R,
$$

and one easily verifies by arguments relying on Fubinis theorem and a countable generator of $\mathcal{S}$ that $(r, s) \mapsto \mathbf{1}_{A_{r}}(s)$ is measurable. Further one can check that $A_{r}$ is $G$-invariant and (7.2) implies that $\nu_{r}\left(A_{r}^{c}\right)=0$. The final step is to set

$$
\bar{\kappa}_{r, s}^{\prime}:=\mathbf{1}_{A_{r}}(s) \bar{\kappa}_{r, s}, \quad s \in S, r \in R
$$

Then by invariance of $A_{r}$ and (7.3)

$$
\bar{\kappa}_{r, g s}^{\prime}(A)=\bar{\kappa}_{r, s}^{\prime}\left(\theta_{g}^{-1} A\right), \quad g \in G, s \in S, A \in \mathcal{T}, r \in R,
$$

and since $\bar{\kappa}_{r, s}^{\prime}=\bar{\kappa}_{r, s}=\kappa_{r, s}, \nu_{r}$-a.e. $s \in S$, the required disintegrations

$$
M_{r}=\nu_{r} \otimes \bar{\kappa}_{r}^{\prime}, \quad r \in R,
$$

hold indeed.

By means of Lemma 7.2 we are now able to prove Theorem 2.1:

Proof of Theorem 2.1: Consider the kernel

$$
M_{s}:=\int \mathbf{1}\{(g s, g) \in \cdot\} \lambda(d g), \quad s \in S
$$


from $S$ to $S \times G$ which is clearly measurably $\sigma$-finite by properness and has the property that every $M_{s}$ is a jointly $G$-invariant measure on $S \times G$. Further it is clear that $\mu_{s}:=$ $\lambda \circ \pi_{s}^{-1}=M_{s}(\cdot \times G)$ and since the $\mu_{s}$ are $\sigma$-finite $G$-invariant measures we may apply Lemma 7.2 with $R:=S, T:=G$ and $\nu_{s}:=\mu_{s}$ to the kernel $M$ to obtain a kernel $\kappa$ from $S \times S$ to $G$ such that (2.4) and the covariance property $(i)$ are fulfilled. Remains to show that $\kappa$ fulfills $(i i),(i i i)$ : For $(i i)$ note that for $s \in S$ by $(2.4)$

$$
\iint \mathbf{1}\{g s \neq t\} \kappa_{s, t}(d g) \mu_{s}(d t)=\int \mathbf{1}\{g s \neq g s\} \lambda(d g)=0 .
$$

This means that

$$
\kappa_{s, t}\left(G_{s, t}^{c}\right)=0, \quad \mu_{s} \text { a.e. } t \in S, s \in S,
$$

and since $\mu_{s} \neq 0$ for each $s \in S$ we may pick some $t \in G s$ such that $\kappa_{s, t}\left(G_{s, t}^{c}\right)=0$ holds. But then by $(i) \kappa_{s, t}\left(G_{s, t}^{c}\right)=0$ for all $t \in G s$. For $(i i i)$ choose $k$ as in (2.3) and note that setting $f(t, g):=k(t)$ in (2.4) yields

$$
\mu_{s} k=\iint k(t) \kappa_{s, t}(G) \mu_{s}(d t)=\kappa_{s, s}(G) \mu_{s} k, \quad s \in S,
$$

where we applied $(i)$ in the last step and which implies $\kappa_{s, s}(G)=1=\kappa_{s, t}(G)$ for $t \in G s$ again by $(i)$.

In applications if some given operation is proper it is usually not hard to determine a suitable partition or simultanously $\mu_{s}$-integrable function $k>0$ on $S$ and hence to actually prove properness. Conversely if this fails it can be hard to prove that a given operation is not proper. The kernel $\kappa$ now gives a tool that enables us to reject properness in certain cases. Say that a subset $L \subset G$ is locally closed if it is the intersection of an open and a closed set. It is well known that such sets inherit local-compactness from $G$.

Corollary 7.3. Let $G$ operate properly on the Borel space $S$ such that $G_{s, s}$ is locally closed in $G$ for all $s \in S$. Then $G_{s, s}$ is compact in $G$ for all $s \in S$.

Proof. The assumption that $G_{s, s}$ is locally closed implies that $G_{s, s}$ is a locally compact subgroup of $G$ and for each $s$ we may choose some left Haar measure $\lambda_{s}$ on $G_{s, s}$. Consider the kernel $\kappa$ from Theorem 2.1. $\kappa_{s, s}$ is concentrated on $G_{s, s}$ and for any $g \in G_{s, s}$ we have by covariance

$$
\kappa_{s, s} \circ \theta_{g}^{-1}=\kappa_{s, g s}=\kappa_{s, s} .
$$

The uniqueness theorem in [10] (Corollary 2.6) now implies $\lambda_{s}=c \cdot \kappa_{s, s}$ hence it is finite which implies compactness of $G_{s, s}$.

Note: Some of the main results of this paper were presented in the workshop "New Perspectives in Stochastic Geometry" at the Mathematisches Forschungsinstitut Oberwolfach, October 2008.

\section{References}

[1] Baccelli, F. and Brémaud, P. (2003). Elements of Queueing Theory. Springer, Berlin. 
[2] Benjamini I., Lyons R., Peres Y., Schramm O. (1999). Group-invariant percolation on graphs. Geom. Funct. Anal. 9, 29-66.

[3] Benjamini I., Schramm O. (2001). Percolation in the hyperbolic plane. J. Amer. Math. Soc. 14, 487-507.

[4] Daley, D.J. and Vere-Jones, D. (2008). An Introduction to the Theory of Point Processes. 2nd ed., Springer, New York.

[5] Dellacherie, C. and Meyer, P.-A. (1975). Probabilités et potentiel. Hermann, Paris.

[6] Gentner, D. and Last, G. (2009). Some mean value formulae for partially stationary tessellations of manifolds. in preparation.

[7] Häggström, O. (1997). Infinite clusters in dependent automorphism invariant percolation on trees. Ann. Probab. 25, 1423-1436.

[8] Kallenberg, O. (1983). Random Measures. Akademie-Verlag Berlin and Academic Press, London.

[9] Kallenberg, O. (2002). Foundations of Modern Probability. Second Edition, Springer, New York.

[10] Kallenberg, O. (2007). Invariant measures and disintegrations with applications to Palm and related kernels. Probab. Th. Rel. Fields 139, 285-310.

[11] Last, G. (2008). Modern random measures: Palm theory and related models. in: New Perspectives in Stochastic Geometry, eds. W. Kendall and I. Molchanov, Clarendon Press, Oxford, to appear.

[12] LASt, G. (2008). Stationary random measures on homogeneous spaces. submitted for publication.

[13] Last, G. and Thorisson, H. (2008). Invariant transports of stationary random measures and mass-stationarity. to appear in Annals of Probability.

[14] Lyons, R. with Peres, Y. (2008). Probability on Trees and Networks. Cambridge University Press. In preparation. Current version available at http://mypage.iu.edu/ ${ }^{\text {rdlyons/. }}$

[15] Matthes, K., Kerstan, J. and Mecke, J. (1978). Infinitely Divisible Point Processes. Wiley, Chichester.

[16] Mecke, J. (1967). Stationäre zufällige Maße auf lokalkompakten Abelschen Gruppen. Z. Wahrsch. verw. Gebiete 9, 36-58.

[17] Nachbin, L. (1965). The Haar Integral. D. van Nostrand Company, Inc., Princeton.

[18] Neveu, J. (1977). Processus ponctuels. École d'Eté de Probabilités de Saint-Flour VI. Lecture Notes in Mathematics 598, 249-445, Springer, Berlin. 
[19] Rother, W. and Zähle, M. (1990). Palm distributions in homogeneous spaces. Math. Nachr. 149, 255-263.

[20] Schneider, R. and Weil, W. (2008). Stochastic and Integral Geometry. Springer, Berlin.

[21] Tortrat, A. (1969). Sur les mesures alatoires dans les groupes non abéliens. Annales de l'institut Henri Poincaré (B) 5, 31-47. 